\title{
Effects of sodium pyruvate supplementation on repeated sprint exercise performance and recovery in male college soccer players: a randomized controlled trial
}

\author{
Yan-Ping Yang ${ }^{1}$, Jun-Qiang Qiu ${ }^{1}$, Meng-Yue Wang ${ }^{1}$, Lin Feng ${ }^{1}$, Dan Luo ${ }^{1}$, Rui-Rui Gao ${ }^{1}$, \\ Fang-Qiang Zhou ${ }^{2}$, Kai-Xuan Che ${ }^{1}$ \\ ${ }^{1}$ Department of Exercise Biochemistry, Exercise Science School, Beijing Sport University, Beijing, China; ${ }^{2}$ Division of Pharmaceutical Research and \\ Development, Shanghai Sandai Pharmaceutical R\&D Co. Ltd., Shanghai, China \\ Contributions: (I) Conception and design: JQ Qiu, YP Yang, FQ Zhou; (II) Administrative support: JQ Qiu; (III) Provision of study materials or \\ patients: JQ Qiu; (IV) Collection and assembly of data: YP Yang, MY Wang; (V) Data analysis and interpretation: JQ Qiu, YP Yang, FQ Zhou; (VI) \\ Manuscript writing: All authors; (VII) Final approval of manuscript: All authors. \\ Correspondence to: Jun-Qiang Qiu. Department of Exercise Biochemistry, Exercise Science School, Beijing Sport University, Beijing 100084, China. \\ Email: qiujunqiang@bsu.edu.cn.
}

\begin{abstract}
Background: Sodium pyruvate (PYR) has been reported to improve aerobic metabolism and attenuate metabolic acidosis. Aerobic capacity and the ability to remove hydrogen ions affect the recovery from repeated high intensity activities. However, the effects of PYR supplementation on repeated sprint exercise (RSE) performance have not been elucidated. This study explored the effects of PYR ingestion on RSE ability and recovery.

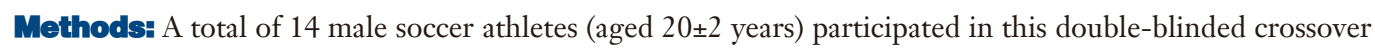
study. The subjects completed two experimental sessions after randomized ingestion of either PYR or the maltodextrin placebo (PLA) for 1 week. At each session, participants completed high-intensity interval exercise (HIIE) and RSE 60 minutes after supplementation. Additionally, acid-base parameters in venous blood, energy system contributions, and power output were assessed.
\end{abstract}

Results: Compared to PLA, PYR supplementation significantly increased the relative peak power output (PPO) of the first $(\mathrm{P}=0.034)$ and fifth $(\mathrm{P}=0.043)$ sprints, and the relative mean power output $(\mathrm{MPO})$ of the fifth sprint $(\mathrm{P}=0.026)$. In addition, the mean PPO $(\mathrm{P}=0.031)$ and MPO $(\mathrm{P}=0.033)$ of sprints $1-6$ were significantly elevated after PYR supplementation. After PYR administration, the phosphagen energy system [adenosine triphosphate (ATP)-phosphocreatine $(\mathrm{PCr})$ ] resynthesis of the fourth $(\mathrm{P}=0.034)$ and the overall recovery periods during $\mathrm{HIIE}(\mathrm{P}=0.029)$ were higher than PLA administration. Additionally, the ATP$\mathrm{PCr}$ resynthesis of the first $(\mathrm{P}=0.033)$ and fifth $(\mathrm{P}=0.019)$ recovery periods, and the mean of the six recovery periods during $\mathrm{RSE}(\mathrm{P}=0.041)$ were increased in the PYR group compared to the PLA group. Furthermore, participants on the PYR regimen had higher blood $\mathrm{pH}, \mathrm{HCO}_{3}^{-}$, and base excess at pre-HIIE, post-HIIE, and pre-RSE (all $\mathrm{P}<0.05$ ) compared to participants receiving PLA.

Conclusions: PYR supplementation enhanced RSE performance, and the improvement may be attributed to accelerated restoration of the acid-base balance and ATP-PCr regeneration.

Trial Registration: Chinese Clinical Trial Registry ChiCTR2100053936.

Keywords: Exercise-induced metabolic acidosis; repeated sprint exercise (RSE); energy metabolism; performance; sodium pyruvate (PYR)

^ ORCID: Jun-Qiang Qiu, 0000-0001-8313-9216; Fang-Qiang Zhou, 0000-0003-1967-6943. 
Submitted Nov 29, 2021. Accepted for publication Jan 20, 2022.

doi: 10.21037/apm-21-3862

View this article at: https://dx.doi.org/10.21037/apm-21-3862

\section{Introduction}

Typical characteristics of team sports include multiple sets of high-intensity exercise and sprints interspersed with short-term recovery sessions, and these exercise bouts usually last for several hours (1). Repeated sprint exercise (RSE) is generally considered to be the determining part to maintain the possession and shooting rates, despite the fact that sprinting activities only account for $10 \%$ of the total distance of team sports (2). However, during highintensity or sprint exercises, the rate of hydrogen ion $\left(\mathrm{H}^{+}\right)$ generation by non-mitochondrial adenosine triphosphate (ATP) far exceeds the rate of neutralization by aerobic ATP (3). Some intracellular $\mathrm{H}^{+}$is transported to the blood, where $\mathrm{H}^{+}$is mainly neutralized by bicarbonate $\left(\mathrm{HCO}_{3}^{-}\right)$(4). With repeat exercise, the increased accumulation of $\mathrm{H}^{+}$in skeletal muscles and the blood is concomitant with a disturbance of the acid-base balance [i.e., decrease in blood $\mathrm{pH}, \mathrm{HCO}_{3}^{-}$, and base excess $(\mathrm{BE})](3,5)$. After bouts of sprinting in the first half of a soccer match, muscle $\mathrm{pH}$ was found to drop to 6.96 compared with 7.24 at rest (5). In addition, significant differences in blood $\mathrm{pH}(7.41-7.25)$ and $\mathrm{HCO}_{3}{ }^{-}$ (24.8-13.6 mmol/L) were observed before and after a team sports game (6). This alteration has been described as exercise-induced metabolic acidosis and is believed to cause fatigue by impairing glycolytic, phosphagen energy [ATPphosphocreatine (PCr)] systems metabolism, and muscle contraction during exercise (7). Additionally, the majority of energy required for sprints is obtained from hydrolysis of the ATP-PCr system. Multiple sprints, however, lead to depletion of PCr which will negatively affect RSE ability $(8,9)$. Throughout a game, RSE capacity of high or ordinary level athletes will gradually decrease $(7,10)$. The ability to recover from these exercise bouts and maintain RSE performance is generally considered to be a crucial factor in winning team sports competitions (2). Interventions that promote ATP-PCr resynthesis and acid-base balance recovery may be therefore beneficial in team sports.

Since the resynthesis of $\mathrm{PCr}$ (11) and the removal of intracellular $\mathrm{H}^{+}$(3) during recovery sessions rely on aerobic metabolism, the RSE ability may be aided by aerobic capacity. Nutrition supplementation strategies including alkaline buffers (such as sodium bicarbonate and $\beta$-alanine) are known to neutralize intracellular or extracellular $\mathrm{H}^{+}$and alleviate the inhibition of glycolysis rate-limiting enzymes, but only improve single sprints or RSE performance by about $2-3 \%(12,13)$. However, co-ingestion of creatine and sodium bicarbonate has been shown to increase both skeletal muscle $\mathrm{PCr}$ content and acid-base recovery, which is more effective in improving RSE performance (14). Therefore, nutritional strategies to improve aerobic metabolic capacity during recovery periods may be beneficial for enhancing RSE performance.

Pyruvate is an important metabolite of glucose and a key metabolic substrate for mitochondrial oxidative metabolism (15). Evidence in numerous preclinical studies suggested that exogenous sodium pyruvate (PYR) supplementation activates pyruvate dehydrogenase $(\mathrm{PDH})$, preserves mitochondrial tricarboxylic acid cycle flux and aerobic metabolism, and can effectively correct metabolic acidosis $(16,17)$. Previous studies found that the blood $\mathrm{pH}$ values of the PYR group were higher than the control group after resuscitation in a model of hemorrhagic shock $(18,19)$. Petrat et al. found that PYR could increase blood $\mathrm{pH}$ and $\mathrm{BE}$ after reperfusion in rat model of severe intestinal ischemia-reperfusion injury (20). More importantly, it was indicated that PYR increased $\mathrm{pH}$ intracellularly in isolated failing human myocardium and reduced $\left(\mathrm{NH}_{4} \mathrm{Cl}\right)$-induced hyperchloremic acidosis in rat model $(21,22)$. Furthermore, Olek et al. found that acute PYR intake increased blood $\mathrm{pH}, \mathrm{HCO}_{3}^{-}$, and $\mathrm{BE}$ at rest in healthy adults (23), but these effects were not observed following 6 minutes of highintensity exercise (24). Two other studies demonstrated that ingestion of PYR for 1 or 2 weeks had no effect on the time to exhaustion during intense exercise (25) or critical power cycle ergometer test performance (26). However, the timeto-exhaustion protocols used in these latter studies may have a high coefficient of variation and are thus not sufficiently accurate for evaluating the effects of PYR in exercise (27). Despite the positive effects of PYR supplementation on aerobic metabolism and acidosis, to date, there have been no studies investigating PYR supplementation on acidbase balance and energy metabolism during high-intensity interval exercise (HIIE) and RSE. Indeed, studies have demonstrated that a 7-day PYR supplementation regimen may have beneficial effects on glucose metabolism during 
high-intensity exercise (28). In addition, 1 week of PYR supplementation may be effective in promoting aerobic metabolism and attenuating acidosis, thereby promoting RSE performance. Therefore, further studies are warranted to explore the efficacy of PYR supplementation for energy and $\mathrm{H}^{+}$metabolism during exercise so as to provide novel nutritional strategies to enhance RSE performance.

This current investigation examined the effects of 1 week of PYR intake on RSE performance and recovery in male soccer players. We hypothesized that PYR supplementation for 1 week would enhance ATP-PCr regeneration and acidbase recovery, leading to an increase in RSE performance. We present the following article in accordance with the CONSORT reporting checklist (available at https://apm. amegroups.com/article/view/10.21037/apm-21-3862/rc).

\section{Methods}

\section{Participants}

This is a randomized, double-blinded, crossover trial with a 1:1 allocation ratio. To avoid potential menstrual cycle effects on energy metabolism, only male participants were enrolled (29). Participants were excluded if they were suffering from hypertension, diabetes, cardiovascular or metabolic diseases; if they had taken cigarettes and/or alcohol; and if they had taken buffering agents within the previous 6 months and throughout this study. The inclusion criteria included the following: (I) participants aged 18-24 years; (II) BMI ranged from 18.5 to $23.9 \mathrm{~kg} / \mathrm{m}^{2}$; and (III) participants had at least 5 years of experience in soccer training with $\mathrm{VO}_{2 \max }$ over $50 \mathrm{~mL} / \mathrm{kg} /$ minute. The study protocol was approved by the Internal Review Board of the Beijing Sport University (No. 2020057H). All procedures performed in this study involving human participants were in accordance with the Declaration of Helsinki (as revised in 2013). Participants were informed of the study aims, possible risks, and signed an informed consent form.

\section{Experimental design}

Participants visited the laboratory on 3 separate sessions, including 1 familiarization session and 2 experimental sessions, each session separated by 14 days. At the first visit, personal information, anthropometric measurements (height and body composition), and graded cycling exercise test (GXT) results were collated. All participants completed the exercise protocol familiarization session. After a 7-day washout period, each participant was then assigned to ingest either $0.1 \mathrm{~g} / \mathrm{kg} / \mathrm{d}$ of PYR or the maltodextrin placebo (PLA) in the following 7 days in a counterbalanced randomized design (i.e., 7 participants were supplemented with PYR and 7 participants were administered the PLA).

During the second and third visits (i.e., on the seventh day of supplementation), 45 minutes following the intake of either PYR or PLA, participants completed a 15-minute resting oxygen uptake test. After a 5-minute warmup, a high-intensity interval exercise (HIIE) cycling test was performed, followed by a 6-minute post-exercise oxygen consumption (EPOC) assessment. Participants undertook the RSE test 4 minutes after the EPOC test.

Oxygen uptake was measured breath-by-breath during GXT, 15 minutes before HIIE, during HIIE, 6 minutes after HIIE, and during RSE, using a portable gas analysis system (Cortex Metamax 3B, CORTEX Biophysik, Leipzig, Germany). Participants completed the oxygen uptake test 15 minutes before and 6 minutes after HIIE in a sitting position. Resting oxygen uptake was taken as the average of the last 10 minutes of data (30). Before each test, the gas analyzer was calibrated according to the instructions of the manufacturer.

Before each visit, all participants visited the laboratory 2 hours after a meal and avoided intense exercise and coffee for 24 hours. The diets for the 24 hours prior to the first visit were recorded. Participants were required to replicate the same diet as accurately as possible 24 hours before the next visits. All participants performed all tests at the same time of day and in the same laboratory environment.

\section{Preliminary assessment and familiarization}

At the first laboratory visit, the participant's height and body composition was measured with a calibrated electronic scale (GMCS-SGJ3, Jianmin, Beijing, China) and a multifrequency bioelectrical impedance measurement device (Inbody 230, Biospace, Seoul, Korea), respectively. Thereafter, each participant performed GXT on an electromagnetically braked cycle ergometer (EC 3000e, CUSTO Med, Ottobrunn, Germany) to determine peak power $\left(\mathrm{W}_{\max }\right)$ and $\mathrm{VO}_{2 \max }$. After adjusting the seat, participants warmed up for 3 minutes at $50 \mathrm{~W}$, the load was then increased by $30 \mathrm{~W} /$ minute with pedal frequency maintained at around 75-80 rpm. During the test, Polar V800 (Polar Electro Oy, Oulu, Finland) was used to measure the heart rate. The standards for exhaustion included at least 3 of the following criteria: (I) failure to 
maintain $75 \mathrm{rpm}$ for 5 seconds; (II) oxygen uptake $\left(\mathrm{VO}_{2}\right)$ changes $\leq 2.1 \mathrm{~mL} / \mathrm{kg} /$ minute in 2 consecutive workloads; (III) respiratory exchange rate $\left(\mathrm{VCO}_{2} / \mathrm{VO}_{2}\right) \geq 1.10$; and (IV) maximal heart rate $\geq 90 \%$ of the predicted maximal value. $\mathrm{VO}_{2 \max }$ is the average oxygen consumption within the final 30 seconds (31). $W_{\max }$ is determined as the load of the last completed stage plus the fraction of time spent in the final uncompleted stage multiplied by $30 \mathrm{~W}$ (32). After a short rest period, all participants completed familiarization with the experimental exercise protocol. Familiarization sessions included performing a HIIE and an RSE. All familiarization protocols were designed to eliminate the effects of learning and training.

\section{Experimental trials}

\section{Supplementation protocol}

High quality PYR ( $\geq 99.0 \%$ pure PYR, Lianlu industrial Co., Ltd., Shanghai, China) and maltodextrin were randomly packaged into capsule A or B by an independent staff. The capsules were similar in appearance, size, and weight. Supplementation with PYR in humans can result in significant blood alkalinization within 1-2 hours (23). Studies have suggested that supplementation with multiple smaller doses may prevent gastrointestinal intolerance (33). Therefore, for the first 6 days, participants took $0.1 \mathrm{~g} / \mathrm{kg} / \mathrm{d}$ PYR or maltodextrin at $25 \%$ dose after breakfast, $25 \%$ after lunch, $25 \%$ after dinner, and $25 \%$ before bed. On the $7^{\text {th }}$ day, the capsules were all ingested at once $(23,25)$. Each 1-day dose was given in a separate plastic bag labeled with a date, and participants were required to consume supplements on time and provide videos. Participants who took capsule A were switched over to capsule B and vice versa during the test. During the study, participants and test personnel were blind and reported similar appearance and taste of the PYR and PLA capsules. Furthermore, none of the participants reported gastrointestinal discomfort with either capsule $(23,25)$.

\section{Primary outcome}

\section{HIIE and RSE tests}

HIIE was conducted on a cycle (Ergoline Ergoselect 100K, Ergoline, Bitz, Germany). During the test, participants undertook a 5 -minute warm-up at $60 \mathrm{~W}$. Then, the HIIE protocol was performed, consisting of 4 sessions of 1-minute cycling at $110 \%$ of their $W_{\max }$, interspersed with 1-minute recovery periods. Cadence was constant (90-100 rpm) during each high-intensity bout (34).

Following HIIE, participants rested for 10 minutes, and then completed 6 sessions of 6 -second maximal cycling, interspersed with 24 seconds passive recovery on a mechanically-braked Monark cycle ergometer (894E, Monark, Vansbro, Sweden). Each sprint exercise was started with a "3, 2, 1, go" countdown. Once cadence reached $110 \mathrm{rpm}$, the $0.087 \mathrm{kp} / \mathrm{kg}$ body mass load was added to the ergometer, and the 6-second sprint was started (35). All participants were encouraged to exert maximum effort possible during each sprint. The seat height and handlebar position for each participant was adjusted prior to the initial HIIE and RSE tests and remained the same for subsequent tests. The RSE test protocol has been reported to be valid and reliable (36), and has been previously used to measure exercise performance in soccer players (37).

All data were calculated via Monark Anaerobic Testing software (version 3.3.0.0, developed in cooperation with HUR Labs) (38). The software automatically recorded the power output per second and calculated the relative peak power output (PPO) and relative mean power output (MPO) for each sprint. The mean PPO and MPO were calculated by taking the average of $\mathrm{PPO}$ and MPO for sprints $1-6$, respectively (38).

\section{Blood collection and analysis}

Venous blood samples $(1.0 \mathrm{~mL})$ obtained from the ulnar vein at baseline, pre-HIIE, post-HIIE, pre-RSE, and post-RSE were collected in sodium heparin tubes (YA1430, Solarbio, Beijing, China) and immediately assessed for blood $\mathrm{pH}$, $\mathrm{HCO}_{3}^{-}$, and $\mathrm{BE}$ using a blood gas analyzer (Radiometer ABL80, FLEX CO-OX, Willich, Germany) (39).

Capillary blood samples $(10 \mu \mathrm{L})$ taken by finger prick were collected with Biosen capillary tubes (EKF Diagnostics, Barleben, Germany) at baseline, immediately after each bout of HIIE, and at 3, 5, 7, and 10 minutes after HIIE. The samples were used to measure blood lactate concentrations with a lactate analyzer (Biosen C-Line, EKF Diagnostics, Barleben, Germany).

\section{Secondary outcome}

\section{Estimation of energy system contributions}

Contributions of the aerobic energy system were estimated by subtracting the resting oxygen uptake from the oxygen consumption obtained during each $110 \% \mathrm{~W}_{\max }$ bout. All oxygen used during HIIE was converted to energy assuming one liter of oxygen is equal to $20.92 \mathrm{~kJ}(40)$. The lactate 
accumulated during each $110 \% \mathrm{~W}_{\max }$ bout was used to evaluate the contributions of the glycolytic energy system of each bout of HIIE ( $1 \mathrm{mmol} / \mathrm{L}$ of lactate equals to $3 \mathrm{~mL} / \mathrm{kg}$ of oxygen) (40). The ATP-PCr resynthesis were calculated by subtracting resting oxygen from the oxygen consumption obtained during each HIIE and RSE recovery periods, and the fast component of post-exercise oxygen consumption $\left(\mathrm{EPOC}_{\text {fast }}\right)$ accessed during the 6 minutes after HIIE. The $\mathrm{EPOC}_{\text {fast }}$ was determined by the product of the amplitude and time constant of the first exponential decay (OriginPro 8.0, OriginLab, Microcal, Massachusetts, USA) $(41,42)$.

\section{Statistical analysis}

A priori analysis with the $G^{*}$ Power software (version 3.1.9.6, Universitat Kiel, Germany) indicated a required sample size of 13 to detect significant differences in acid-base balance values. The following parameters were applied: repeated measures ANOVA within factors; effect size $(\mathrm{f})=0.24$; $\alpha=0.05$; power $(1-\beta)=0.8$; and correction among repeated measures $=0.5$ (43). Data analyses were performed using SPSS software (version 22.0, SPSS Inc. Chicago, IL, USA) and presented as the mean \pm standard deviation (SD). The Shapiro-Wilk test was used to determine the data normality. Two-way repeated-measures ANOVA was performed to assess the interaction between time and the two groups, and post-hoc analysis was performed using Fisher's least significant difference test. Effect sizes were calculated as partial eta-squared $\left[\eta_{\mathrm{p}}{ }^{2}\right.$; small effect $(0.01-0.059)$, medium effect (0.06-0.139), and large effect $(\geq 0.14)]$. Independent samples $t$-tests were used to measure average PPO and $M P O$, and average contributions of the energy system during HIIE and RSE. Effect sizes were expressed as Cohen's d, where Cohen's $d=0.20-0.49$ indicates a small effect, Cohen's $\mathrm{d}=0.50-0.79$ indicates a medium effect, and Cohen's $d \geq 0.80$ indicates a large effect. The significance level was set at $\mathrm{P}<0.05$.

\section{Results}

Between October and December 2020, 15 national-level male soccer athletes from the China Football College were enrolled in this study. Figure 1 shows the enrollment and follow-up of the participants. One participant dropped out after completing the first visit due to personal reasons. Fourteen athletes completed all the sessions and were included in the data analysis. The baseline characteristics of participants are shown in Table 1.

\section{RSE performance}

Post-hoc analysis showed significant differences between PYR and PLA groups in the PPO of the first sprint $\left(\mathrm{P}=0.034, \eta_{\mathrm{p}}{ }^{2}\right.$ $=0.161)$ and fifth sprints $\left(\mathrm{P}=0.043, \eta_{\mathrm{p}}{ }^{2}=0.149\right)($ Figure $2 A)$. In addition, the MPO of the fifth sprint $\left(\mathrm{P}=0.026, \eta_{\mathrm{p}}{ }^{2}\right.$ $=0.177$ ) was significantly improved after PYR administration compared to PLA administration (Figure $2 B$ ).

The mean PPO $(\mathrm{P}=0.031$, Cohen's $\mathrm{d}=0.86)$ (Figure $2 C$ ) and the mean MPO of the 6 sets of sprints $(\mathrm{P}=0.033$, Cohen's d =0.85) (Figure 2D) were higher after PYR administration compared to PLA administration.

\section{Energy system contributions}

During HIIE, there were no difference between PYR and PLA groups in the aerobic energy system contributions $\left(\mathrm{P}=0.275, \eta_{\mathrm{p}}{ }^{2}=0.046\right)$ (Figure $\left.3 A\right)$. Compared with the PLA group, the ATP-PCr resynthesis of the fourth recovery period $\left(\mathrm{P}=0.034, \eta_{\mathrm{p}}{ }^{2}=0.161\right)$ during HIIE was significantly improved in the PYR group (Figure 3B). For contributions of the glycolytic energy during HIIE, a significant reduction was observed during repeated high-intensity exercise in both groups $\left(\mathrm{P}<0.0001, \eta_{\mathrm{p}}{ }^{2}=0.802\right)$. However, no changes were observed in the contributions of glycolytic energy between PYR and PLA groups $\left(\mathrm{P}=0.410, \eta_{\mathrm{p}}{ }^{2}=0.026\right.$ ) (Figure $3 C$ ).

The total contribution of aerobic energy $(\mathrm{P}=0.275$; Figure $3 D$ ) during HIIE was not changed between the two groups. PYR supplementation enhanced the total ATPPCr resynthesis during HIIE $(\mathrm{P}=0.029$, Cohen's $\mathrm{d}=0.874$; Figure $3 E)$. However, the total contribution of glycolytic energy $(\mathrm{P}=0.411$; Figure $3 F)$ during HIIE did not differ between the two groups.

During RSE, compared with the PLA group, the ATP$\mathrm{PCr}$ resynthesis of the first $\left(\mathrm{P}=0.033, \eta_{\mathrm{p}}{ }^{2}=0.163\right)$ and fifth $\left(\mathrm{P}=0.019, \eta_{\mathrm{p}}{ }^{2}=0.193\right)$ recovery periods were significantly improved in the PYR group (Figure 4A). In addition, the total ATP-PCr resynthesis was significantly improved by PYR than PLA supplement $(\mathrm{P}=0.041$, Cohen's $\mathrm{d}=0.81)$ during RSE (Figure 4B).

\section{Blood gas analysis}

Table 2 shows the changes in blood $\mathrm{pH}, \mathrm{HCO}_{3}^{-}$, and $\mathrm{BE}$ at baseline, during pre- and post-HIIE and RSE. There was no effect of supplementation order on blood $\mathrm{pH}, \mathrm{HCO}_{3}^{-}$, nor $\mathrm{BE}$ at baseline (all $\mathrm{P}>0.05$ ). However, blood $\mathrm{pH}, \mathrm{HCO}_{3}{ }^{-}$, and $\mathrm{BE}$ decreased from baseline to pre-HIIE and post- 


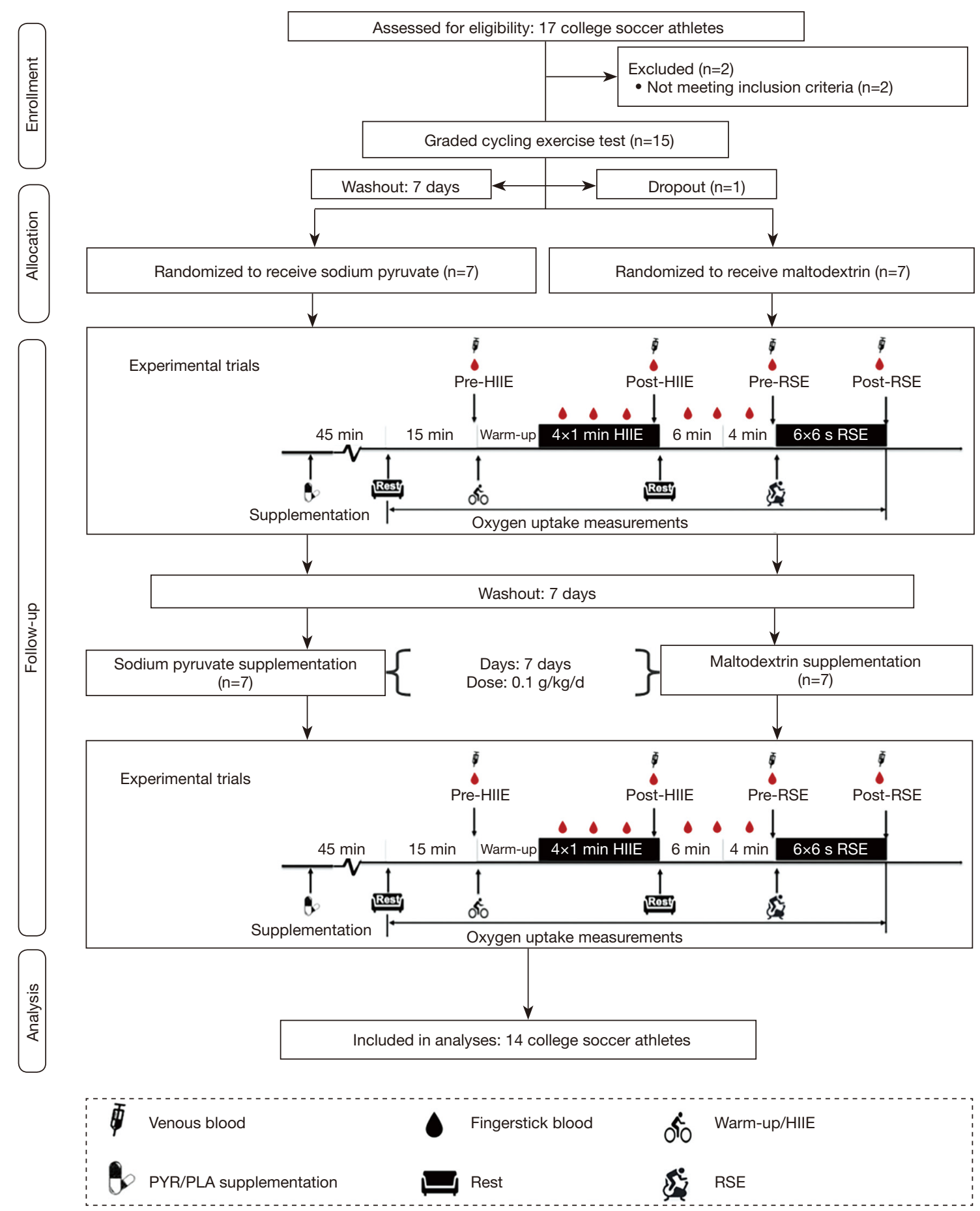

Figure $1 \mathrm{~A}$ timeline of the supplementation and exercise tests. HIIE, high-intensity interval exercise; RSE, repeated sprint exercise; PYR, sodium pyruvate; PLA, placebo. 
Table 1 Baseline characteristics of participants (n=14)

\begin{tabular}{lc}
\hline Characteristics & Value, mean $\pm \mathrm{SD}$ \\
\hline Age (year) & $20 \pm 2$ \\
Height $(\mathrm{cm})$ & $178.3 \pm 6.4$ \\
Weight $(\mathrm{kg})$ & $69.58 \pm 7.22$ \\
Body fat $(\%)$ & $13.11 \pm 3.50$ \\
$\mathrm{VO}_{2 \text { max }}(\mathrm{mL} / \mathrm{kg} / \mathrm{min})$ & $55.93 \pm 5.36$ \\
$\mathrm{~W}_{\text {max }}(\mathrm{W})$ & $298.54 \pm 41.70$ \\
Training year (year) & $9 \pm 3$ \\
\hline
\end{tabular}

$\mathrm{SD}$, standard deviation; $\mathrm{VO}_{2 \max }$, maximal oxygen uptake; $\mathrm{W}_{\max }$, maximal power output.
HIIE levels, as well as from pre-RSE to post-RSE levels in both groups (all $\mathrm{P}<0.05)$. Furthermore, compared with the PLA group, blood $\mathrm{pH}$ was significantly higher at pre-HIIE $\left(\mathrm{P}=0.018, \eta_{\mathrm{p}}{ }^{2}=0.198\right)$, post-HIIE $\left(\mathrm{P}=0.041, \eta_{\mathrm{p}}{ }^{2}=0.15\right)$, and pre-RSE $\left(\mathrm{P}=0.032, \eta_{\mathrm{p}}{ }^{2}=0.165\right)$ following PYR ingestion. Blood $\mathrm{HCO}_{3}^{-}$levels were significantly higher at pre-HIIE $\left(\mathrm{P}=0.002, \eta_{\mathrm{p}}{ }^{2}=0.316\right)$, post-HIIE $\left(\mathrm{P}=0.01, \eta_{\mathrm{p}}{ }^{2}=0.238\right)$, and pre-RSE $\left(\mathrm{P}=0.019, \eta_{\mathrm{p}}{ }^{2}=0.195\right)$ with $\mathrm{PYR}$ supplementation compared to PLA. PYR administration also resulted in higher blood $\mathrm{BE}$ at pre-HIIE $\left(\mathrm{P}=0.002, \eta_{\mathrm{p}}{ }^{2}=0.323\right)$, postHIIE $\left(\mathrm{P}=0.047, \eta_{\mathrm{p}}{ }^{2}=0.143\right)$, pre-RSE $\left(\mathrm{P}=0.036, \eta_{\mathrm{p}}{ }^{2}=0.158\right)$, and post-RSE $\left(\mathrm{P}=0.021, \eta_{\mathrm{p}}{ }^{2}=0.189\right)$ compared to PLA administration.
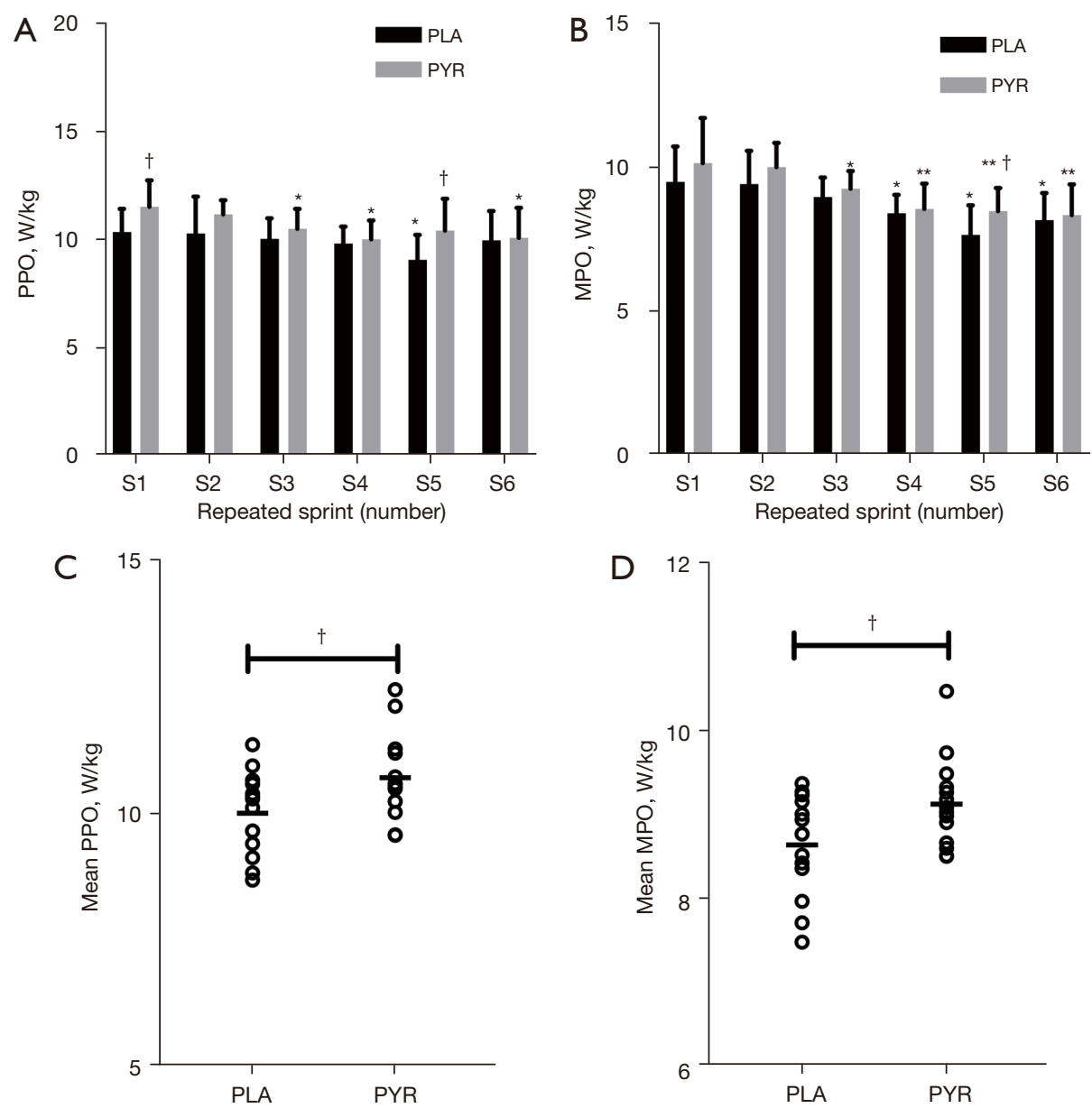

Figure 2 Effects of PYR and PLA supplementation on RSE performance. The PPO (A) and MPO (B) of each sprint. The mean PPO (C) and mean MPO (D) of sprints $1-6 .{ }^{*}, \mathrm{P}<0.05$; ${ }^{* *}, \mathrm{P}<0.01$ vs. S1 of the same group; ${ }^{\dagger}, \mathrm{P}<0.05$ vs. PLA at the same time point. $\mathrm{PPO}$, peak power output; MPO, mean power output; PYR, sodium pyruvate; PLA, placebo; RSE, repeated sprint exercise. 

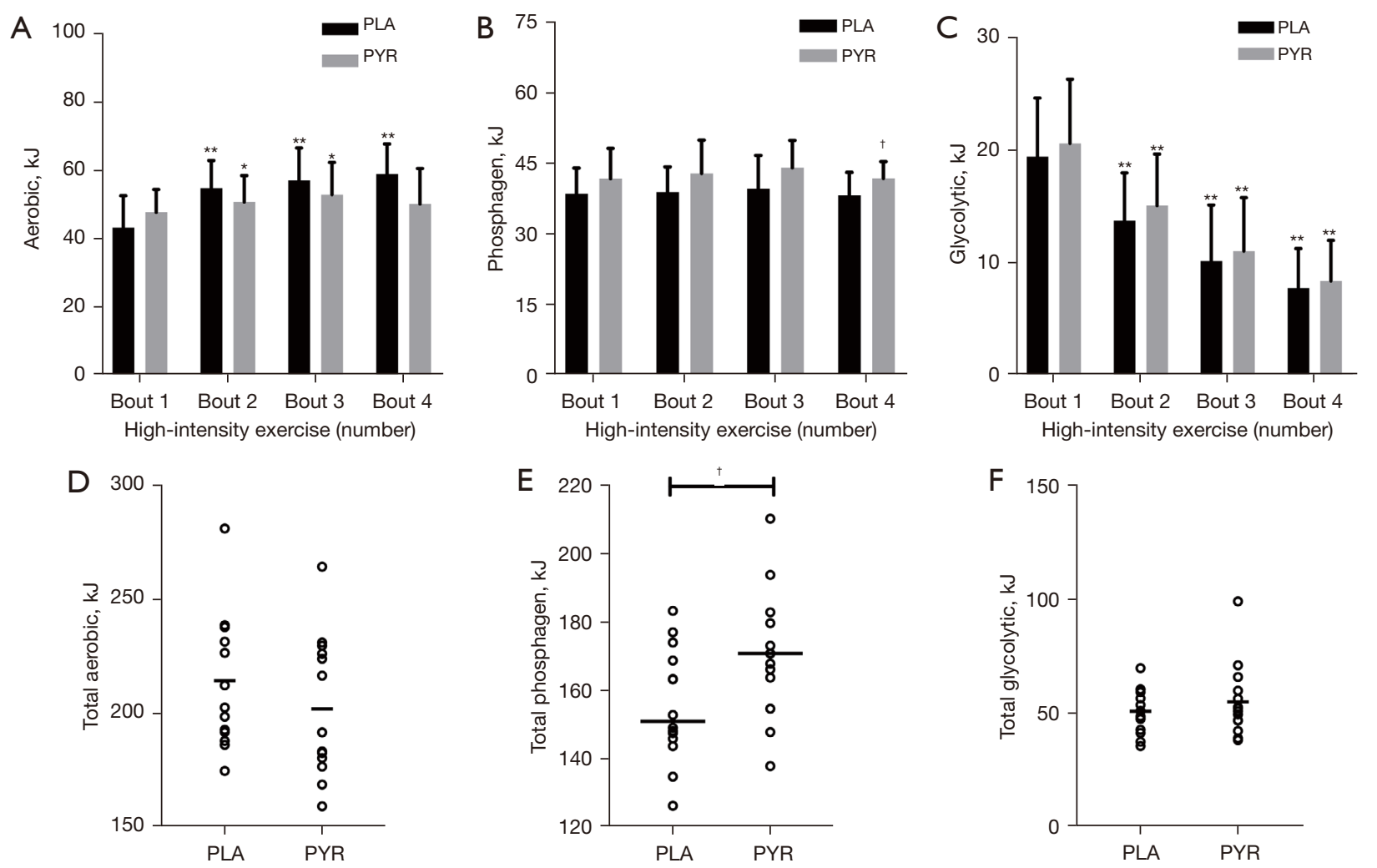

Figure 3 Effects of PYR and PLA supplementation on the contributions of the energy systems. Contributions of the aerobic (A,D) and glycolytic $(\mathrm{C}, \mathrm{F})$ energy systems during HIIE. The phosphagen energy system resynthesis $(\mathrm{B}, \mathrm{E})$ during HIIE. *, $\mathrm{P}<0.05 ;{ }^{* *}, \mathrm{P}<0.01$ vs. bout 1 of the same group; ${ }^{\dagger}, \mathrm{P}<0.05$ vs. PLA at the same time point. PLA, placebo; HIIE, high-intensity interval exercise; PYR, sodium pyruvate.

\section{Discussion}

The main findings of this study suggested that 1 week of PYR ingestion enhanced the power output during RSE. In support of our initial hypothesis, the resynthesis of $\mathrm{PCr}$ and acid-base balance recovery during HIIE and RSE were improved in the PYR group.

Many team sports (such as soccer and basketball) consist of HIIE sessions and repeated bouts of sprints with short recovery (1). A large accumulation of $\mathrm{H}^{+}$and the depletion of skeletal muscle PCr content are evident in subsequent bouts of exercise, leading to fatigue during RSE (8). Consistent with previous findings, we observed that the decline in PPO and MPO was accompanied by the repetition of sprints in both the PYR and PLA groups (44).

In the present study, PYR ingestion elevated the PPO in the first $(+11.41 \%)$ and fifth $(+14.92 \%)$ sprints, and the MPO in the fifth sprint $(+10.72 \%)$. Additionally, the mean PPO $(+7.06 \%)$ and mean MPO $(+5.56 \%)$ of the 6 sets of sprints were significantly improved following
PYR administration compared to PLA administration (Figure 2). Although, previous studies showed that PYR supplementation did not enhance aerobic exercise capacity $(25,26)$, the current study demonstrated that for intense exercise, PYR supplementation resulted in a stronger ergogenic effect on RSE performance compared to other common buffers that only obtained a $2-3 \%$ increase in power output during single sprints or RSE $(12,13)$. Indeed, the stronger ergogenic effect may be attributed to the fact that PYR ingestion promotes phosphagen metabolism and attenuates acidosis during RSE. The beneficial effects of PYR observed in this study confirmed the findings of Sarshin et al. The latter study demonstrated that a combined improvement in PCr content and acid-base balance may be more beneficial for RSE ability (14).

The ergogenic effects of PYR intake may be explained by enhanced aerobic metabolism. Although PCr hydrolysis and anaerobic glycolysis are rapid pathway for ATP production during sprint exercise, the aerobic system plays an increasingly important role in maintaining performance 

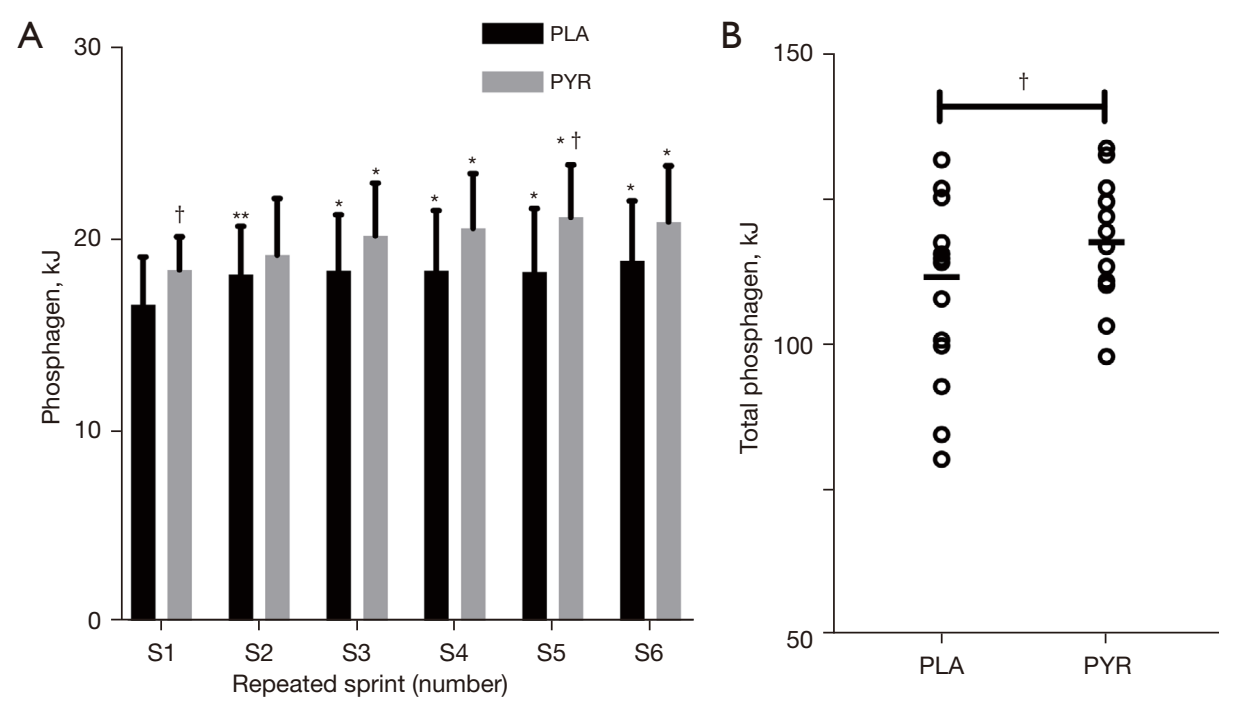

Figure 4 Effects of PYR and PLA supplementation on the phosphagen energy system resynthesis during RSE. (A) The phosphagen energy system resynthesis during each recovery session. (B) Total phosphagen energy system resynthesis of the 6 sets of recovery sessions. *, $\mathrm{P}<0.05$; **, $\mathrm{P}<0.01$ vs. $\mathrm{S} 1$ of the same group; ${ }^{\dagger}, \mathrm{P}<0.05$ vs. PLA at the same time point. PLA, placebo; PYR, sodium pyruvate; RSE, repeated sprint exercise.

Table 2 Comparison of blood $\mathrm{pH}, \mathrm{HCO}_{3}^{-}$, and $\mathrm{BE}$ between the two groups

\begin{tabular}{|c|c|c|c|c|c|c|}
\hline Time point & \multicolumn{2}{|c|}{$\mathrm{pH}$, mean $\pm \mathrm{SD}$} & \multicolumn{2}{|c|}{$\mathrm{HCO}_{3}^{-}(\mathrm{mmol} / \mathrm{L})$, mean $\pm \mathrm{SD}$} & \multicolumn{2}{|c|}{$\mathrm{BE}(\mathrm{mmol} / \mathrm{L})$, mean $\pm \mathrm{SD}$} \\
\hline Baseline & $7.36 \pm 0.03$ & $7.38 \pm 0.04$ & $24.52 \pm 0.96$ & $24.55 \pm 1.15$ & $1.16 \pm 1.30$ & $1.06 \pm 1.15$ \\
\hline Pre-HIIE & $7.37 \pm 0.03$ & $7.40 \pm 0.03^{\dagger}$ & $24.86 \pm 1.03$ & $26.09 \pm 0.82^{\star \star \dagger \dagger}$ & $2.00 \pm 1.11$ & $3.39 \pm 0.86^{\star \star \dagger \dagger}$ \\
\hline Post-HIIE & $7.14 \pm 0.08^{\star \star}$ & $7.20 \pm 0.05^{\star \star \dagger}$ & $12.57 \pm 1.86^{\star \star}$ & $14.56 \pm 1.83^{\star \star \dagger}$ & $-15.61 \pm 4.39^{\star \star}$ & $-12.58 \pm 3.24^{\star \star \dagger}$ \\
\hline Post-RSE & $7.18 \pm 0.07^{\star \star}$ & $7.23 \pm 0.07^{\star \star}$ & $12.52 \pm 1.61^{* *}$ & $14.04 \pm 2.32^{\star *}$ & $-16.56 \pm 3.07^{\star \star}$ & $-13.64 \pm 3.50^{\star \star \dagger}$ \\
\hline
\end{tabular}

**, $\mathrm{P}<0.01$ : significantly different $v s$. baseline of the same group; ${ }^{\dagger}, \mathrm{P}<0.05 ;{ }^{\dagger \dagger}, \mathrm{P}<0.01$ : significantly different $v s$. $\mathrm{PLA}$ at the same time point. SD, standard deviation; BE, base excess; PLA, placebo group; PYR, pyruvate group; HIIE, high-intensity interval exercise; RSE, repeated sprint exercise.

during repeated sprints (38). As shown in previous studies, large amounts of $\mathrm{PCr}$ are consumed in multiple sprints, and short recovery periods may not afford sufficient time for the PCr values to restore to resting levels, thus leading to RSE fatigue (8). In fact, the resynthesis of $\mathrm{PCr}$ is dependent on aerobic metabolism, and therefore, higher levels of aerobic metabolism accelerate ATP-PCr resynthesis during the recovery periods and maintains subsequent sprint ability (45). According to our results, PYR significantly enhanced the ATP-PCr regeneration in the fourth bout of HIIE $\left(+9.39 \%, \eta_{\mathrm{p}}{ }^{2}=0.161\right)$ (Figure $3 B$ ) and increased the total ATP-PCr regeneration during HIIE $(+9.72 \%$, Cohen's d $=0.874)$ (Figure $3 E)$ with large effect sizes. In addition, the ATP-PCr regeneration was improved in the first $(+10.99 \%)$ and fifth $(+15.54 \%)$ (Figure $4 A$ ) recovery periods during RSE. Consistent with the findings of Knott et al, administration of PYR upon reperfusion after cardioplegic arrest could protect mitochondrial enzymes and increase phosphorylation potential and ATP content (46). In addition, although previous studies did not find a positive effect of PYR on improving aerobic metabolism during exercise, Sharma and Zhang showed 
that PYR activated PDH activity and promoted more ATP production by mitochondrial aerobic metabolism $(17,47)$. In summary, as $\mathrm{PCr}$ resynthesis capacity is a limiting factor for RSE performance, it may also account for the greater beneficial effects of pyruvate supplementation on exercise performance in the present study.

Furthermore, the improvement of RSE ability by PYR supplementation may also be related to the increased acidbase recovery capacity in the blood. It has been suggested that the rapid restoration of acid-base balance during exercise reduces the inhibition of energy metabolism and muscle contraction by $\mathrm{H}^{+}(7)$. In the current study, following 1 week of PYR ingestion, blood $\mathrm{pH}$ (+0.03 units), $\mathrm{HCO}_{3}{ }^{-}$ $(+1.23 \mathrm{mmol} / \mathrm{L})$, and $\mathrm{BE}(+1.39 \mathrm{mmol} / \mathrm{L})$ were significantly higher before HIIE in the PYR group compared to the PLA group. Additionally, blood $\mathrm{pH}$ (+0.07 units), $\mathrm{HCO}_{3}{ }^{-}$ $(+2.22 \mathrm{mmol} / \mathrm{L})$, and $\mathrm{BE}(+2.92 \mathrm{mmol} / \mathrm{L})$ were elevated before the start of RSE in the PYR group compared to the PLA group. Although the mechanisms of improved buffering capacity with 1 week of PYR ingestion remain unknown, the enhanced $\mathrm{PCr}$ resynthesis capacity may provide some insight (3). Compared with sodium bicarbonate ingestion (12), PYR supplementation in the present study had a mild alkalization effect on blood $\mathrm{pH}$ and $\mathrm{HCO}_{3}^{-}$, which is consistent with the results observed by Olek et al. (23). Common buffering agents (e.g., sodium bicarbonate) have been shown to maintain glycolytic metabolism during high-intensity exercise primarily through a stronger regulation of acid-base homeostasis (48). In our study, the blood lactate concentrations and glycolytic metabolism did not differ between PYR administration and PLA administration. The possible reason is that the mild regulation of acid-base recovery by PYR supplementation may not be sufficient to alleviate the inhibitory effect of $\mathrm{H}^{+}$accumulation on glycolytic metabolism $(1,12)$. Also, previous studies have similarly found that 1 week of PYR supplementation failed to boost the glycolytic energy supply during high-intensity exercise (25). Nevertheless, the modulation of acid-base balance recovery by PYR may achieve benefits for exercise performance by reducing the inhibition of $\mathrm{PCr}$ resynthesis and muscle contraction through the reduction of $\mathrm{H}^{+}$concentration (7).

This study had some limitations. First, since the differences in muscle anatomy and physiology between males and females, our findings should not be extrapolated to females. Second, as the athletes in this study had a training commitment, we only calculated the contribution of energy systems. Collecting skeletal muscle for assessing intracellular pH, PCr, ATP levels, and PDH activity could provide further insights on the effects of PYR on acid-base homeostasis and aerobic energy metabolism. Additionally, considering the inconvenience of gas metabolism and venous blood collection in the field test, the bicycle task was used in this study. However, there are differences between the bicycle task and field tests, and therefore, it is necessary to extrapolated the results of this study to team sports with caution.

\section{Conclusions}

This study demonstrated that PYR supplementation $(0.1 \mathrm{~g} / \mathrm{kg} / \mathrm{d}$ for 1 week) can improve power output during RSE in male college soccer players. This improvement in exercise performance may result from the accelerated restoration of acid-base balance and increased ATP-PCr resynthesis. However, future research is still warranted to confirm our findings and to elucidate the mechanisms that may be responsible for the ergogenic effects of PYR.

\section{Acknowledgments}

The authors would like to sincerely thank the participants for volunteering to participate in this study.

Funding: Funding was provided by the National Key R\&D Program of China (No. 2018YFC2000600) and the Herbalife Winter Sports Development Fund (No. KBL2019003).

\section{Footnote}

Reporting Checklist: The authors have completed the CONSORT reporting checklist. Available at https://apm. amegroups.com/article/view/10.21037/apm-21-3862/rc

Trial Protocol: Available at https://apm.amegroups.com/ article/view/10.21037/apm-21-3862/tp

Data Sharing Statement: Available at https://apm.amegroups. com/article/view/10.21037/apm-21-3862/dss

Conflicts of Interest: All authors have completed the ICMJE uniform disclosure form (available at https://apm.amegroups. com/article/view/10.21037/apm-21-3862/coif). Dr. FQZ is from Shanghai Sandai Pharmaceutical R\&D Co. Ltd. The 
other authors have no conflicts of interest to declare.

Ethical Statement: The authors are accountable for all aspects of the work in ensuring that questions related to the accuracy or integrity of any part of the work are appropriately investigated and resolved. All procedures performed in this study involving human participants were in accordance with the Declaration of Helsinki (as revised in 2013). The study protocol was approved by the Internal Review Board of the Beijing Sport University (No. 2020057H). All participants signed written informed consent prior to participating in this study.

Open Access Statement: This is an Open Access article distributed in accordance with the Creative Commons Attribution-NonCommercial-NoDerivs 4.0 International License (CC BY-NC-ND 4.0), which permits the noncommercial replication and distribution of the article with the strict proviso that no changes or edits are made and the original work is properly cited (including links to both the formal publication through the relevant DOI and the license). See: https://creativecommons.org/licenses/by-nc-nd/4.0/.

\section{References}

1. Spencer M, Bishop D, Dawson B, et al. Physiological and metabolic responses of repeated-sprint activities:specific to field-based team sports. Sports Med 2005;35:1025-44.

2. Carling C, Bloomfield J, Nelsen L, et al. The role of motion analysis in elite soccer: contemporary performance measurement techniques and work rate data. Sports Med 2008;38:839-62.

3. Robergs RA, Ghiasvand F, Parker D. Biochemistry of exercise-induced metabolic acidosis. Am J Physiol Regul Integr Comp Physiol 2004;287:R502-16.

4. Juel C, Klarskov C, Nielsen JJ, et al. Effect of highintensity intermittent training on lactate and $\mathrm{H}+$ release from human skeletal muscle. Am J Physiol Endocrinol Metab 2004;286:E245-51.

5. Krustrup P, Mohr M, Steensberg A, et al. Muscle and blood metabolites during a soccer game: implications for sprint performance. Med Sci Sports Exerc 2006;38:1165-74.

6. Couderc A, Thomas C, Lacome M, et al. Movement Patterns and Metabolic Responses During an International Rugby Sevens Tournament. Int J Sports Physiol Perform 2017;12:901-7.

7. MacIntosh BR, Holash RJ, Renaud JM. Skeletal muscle fatigue--regulation of excitation-contraction coupling to avoid metabolic catastrophe. J Cell Sci 2012;125:2105-14.

8. Bishop D, Girard O, Mendez-Villanueva A. Repeatedsprint ability - part II: recommendations for training. Sports Med 2011;41:741-56.

9. Atakan MM, Karavelioğlu MB, Harmancı H, et al. Short term creatine loading without weight gain improves sprint, agility and leg strength performance in female futsal players. Science \& Sports 2019;34:321-7.

10. Robergs R, Kennedy D. Metabolic acidosis and fatigue: Where to from here? In: Marino FE. editor. Regulation of fatigue in exercise. USA: Nova Science Publishers, 2011:41-61.

11. Gaitanos GC, Williams C, Boobis LH, et al. Human muscle metabolism during intermittent maximal exercise. J Appl Physiol (1985) 1993;75:712-9.

12. Carr AJ, Hopkins WG, Gore CJ. Effects of acute alkalosis and acidosis on performance: a meta-analysis. Sports Med 2011;41:801-14.

13. Culbertson JY, Kreider RB, Greenwood M, et al. Effects of beta-alanine on muscle carnosine and exercise performance: a review of the current literature. Nutrients 2010;2:75-98.

14. Sarshin A, Fallahi V, Forbes SC, et al. Short-term coingestion of creatine and sodium bicarbonate improves anaerobic performance in trained taekwondo athletes. J Int Soc Sports Nutr 2021;18:10.

15. Zhou FQ. Pyruvate in the correction of intracellular acidosis: a metabolic basis as a novel superior buffer. Am J Nephrol 2005;25:55-63.

16. Kukimoto-Niino $M$, Tokmakov A, Terada T, et al. Inhibitor-bound structures of human pyruvate dehydrogenase kinase 4. Acta Crystallogr D Biol Crystallogr 2011;67:763-73.

17. Sharma P, Walsh KT, Kerr-Knott KA, et al. Pyruvate modulates hepatic mitochondrial functions and reduces apoptosis indicators during hemorrhagic shock in rats. Anesthesiology 2005;103:65-73.

18. Hu S, Bai XD, Liu XQ, et al. Pyruvate Ringer's solution corrects lactic acidosis and prolongs survival during hemorrhagic shock in rats. J Emerg Med 2013;45:885-93.

19. Flaherty DC, Hoxha B, Sun J, et al. Pyruvate-fortified fluid resuscitation improves hemodynamic stability while suppressing systemic inflammation and myocardial oxidative stress after hemorrhagic shock. Mil Med 2010;175:166-72.

20. Petrat F, Rönn T, de Groot H. Protection by pyruvate 
infusion in a rat model of severe intestinal ischemiareperfusion injury. J Surg Res 2011;167:e93-e101.

21. Hasenfuss G, Maier LS, Hermann HP, et al. Influence of pyruvate on contractile performance and $\mathrm{Ca}(2+)$ cycling in isolated failing human myocardium. Circulation 2002;105:194-9.

22. Yang J, Zhao JX, Wang Y, et al. Effects of sodium pyruvate on ameliorating metabolic acidosis. Artif Cells Nanomed Biotechnol 2016;44:48-55.

23. Olek RA, Luszczyk M, Kujach S, et al. Single pyruvate intake induces blood alkalization and modification of resting metabolism in humans. Nutrition 2015;31:466-74.

24. Olek RA, Kujach S, Wnuk D, et al. Single sodium pyruvate ingestion modifies blood acid-base status and post-exercise lactate concentration in humans. Nutrients 2014;6:1981-92.

25. Morrison MA, Spriet LL, Dyck DJ. Pyruvate ingestion for 7 days does not improve aerobic performance in welltrained individuals. J Appl Physiol (1985) 2000;89:549-56.

26. Ebersole KT, Stout JR, Eckerson JM, et al. The effect of pyruvate supplementation on critical power. J Strength Cond Res 2000;14:132-4.

27. Currell K, Jeukendrup AE. Validity, reliability and sensitivity of measures of sporting performance. Sports Med 2008;38:297-316.

28. Stanko RT, Robertson RJ, Galbreath RW, et al. Enhanced leg exercise endurance with a high-carbohydrate diet and dihydroxyacetone and pyruvate. J Appl Physiol (1985) 1990;69:1651-6.

29. Isacco L, Duché P, Boisseau N. Influence of hormonal status on substrate utilization at rest and during exercise in the female population. Sports Med 2012;42:327-42.

30. Camps SG, Wang NX, Tan WS, et al. Estimation of basal metabolic rate in Chinese: are the current prediction equations applicable? Nutr J 2016;15:79.

31. Edvardsen E, Hem E, Anderssen SA. End criteria for reaching maximal oxygen uptake must be strict and adjusted to sex and age: a cross-sectional study. PLoS One 2014;9:e85276.

32. Wang J, Qiu J, Yi L, et al. Effect of sodium bicarbonate ingestion during 6 weeks of HIIT on anaerobic performance of college students. J Int Soc Sports Nutr 2019;16:18.

33. Grgic J, Pedisic Z, Saunders B, et al. International Society of Sports Nutrition position stand: sodium bicarbonate and exercise performance. J Int Soc Sports Nutr 2021;18:61.

34. da Silva RP, de Oliveira LF, Saunders B, et al. Effects of $\beta$-alanine and sodium bicarbonate supplementation on the estimated energy system contribution during high-intensity intermittent exercise. Amino Acids 2019;51:83-96.

35. Bar-Or O. The Wingate anaerobic test. An update on methodology, reliability and validity. Sports Med 1987;4:381-94.

36. McGawley K, Bishop D. Reliability of a 5 x 6-s maximal cycling repeated-sprint test in trained female team-sport athletes. Eur J Appl Physiol 2006;98:383-93.

37. Billaut F, Smith K. Prolonged repeated-sprint ability is related to arterial O2 desaturation in men. Int J Sports Physiol Perform 2010;5:197-209.

38. Girard O, Mendez-Villanueva A, Bishop D. Repeatedsprint ability - part I: factors contributing to fatigue. Sports Med 2011;41:673-94.

39. Kurtz I, Kraut J, Ornekian V, et al. Acid-base analysis: a critique of the Stewart and bicarbonatecentered approaches. Am J Physiol Renal Physiol 2008;294:F1009-31.

40. di Prampero PE, Ferretti G. The energetics of anaerobic muscle metabolism: a reappraisal of older and recent concepts. Respir Physiol 1999;118:103-15.

41. Ozyener F, Rossiter HB, Ward SA, et al. Influence of exercise intensity on the on- and off-transient kinetics of pulmonary oxygen uptake in humans. J Physiol 2001;533:891-902.

42. Panissa VLG, Fukuda DH, Caldeira RS, et al. Is Oxygen Uptake Measurement Enough to Estimate Energy Expenditure During High-Intensity Intermittent Exercise? Quantification of Anaerobic Contribution by Different Methods. Front Physiol 2018;9:868.

43. Beck TW. The importance of a priori sample size estimation in strength and conditioning research. J Strength Cond Res 2013;27:2323-37.

44. Crisafulli DL, Buddhadev HH, Brilla LR, et al. Creatineelectrolyte supplementation improves repeated sprint cycling performance: A double blind randomized control study. J Int Soc Sports Nutr 2018;15:21.

45. Cooper R, Naclerio F, Allgrove J, et al. Creatine supplementation with specific view to exercise/sports performance: an update. J Int Soc Sports Nutr 2012;9:33.

46. Knott EM, Sun J, Lei Y, et al. Pyruvate mitigates oxidative stress during reperfusion of cardioplegia-arrested myocardium. Ann Thorac Surg 2006;81:928-34.

47. Zhang XM, Deng H, Tong JD, et al. Pyruvate-Enriched Oral Rehydration Solution Improves Glucometabolic Disorders in the Kidneys of Diabetic db/db Mice. J 
Diabetes Res 2020;2020:2817972.

48. Lancha Junior AH, Painelli Vde S, Saunders B, et al. Nutritional Strategies to Modulate Intracellular and Extracellular Buffering Capacity During High-Intensity
Exercise. Sports Med 2015;45 Suppl 1:S71-81.

(English Language Editor: J. Teoh)

Cite this article as: Yang YP, Qiu JQ, Wang MY, Feng L, Luo D, Gao RR, Zhou FQ, Che KX. Effects of sodium pyruvate supplementation on repeated sprint exercise performance and recovery in male college soccer players: a randomized controlled trial. Ann Palliat Med 2022;11(2):598-610. doi: 10.21037/apm-213862 